\title{
PRÁCTICAS DE LA FILOSOFÍA DISCIPLINAR CHILENA: EL FONDECYT REGULAR ${ }^{1}$
}

\author{
Practices of the chilean disciplinar philosophy: the regular \\ FONDECYT
}

\author{
Matías Silva Rojas $\left(^{*}\right)$ \\ RESUMEN
}

El artículo pretende hacer un análisis de las prácticas académicas efectuadas por los filósofos que en Chile postulan a la adjudicación de fondos concursables. El objetivo es poner de manifiesto la intencionalidad del desarrollo de investigaciones disciplinares como forma de mantener vigente el ejercicio de la profesión. De este modo, el análisis centra su mirada en una de las pautas de evaluación del concurso Fondo Nacional de Desarrollo Científico y Tecnológico (FONDECYT) perteneciente al área de Filosofía. Con ello, se busca precisar el criterio utilizado para producir y reproducir un tipo específico de filosofía siguiendo el ideal moderno sobre la misma: hacer filosofía como ciencia.

\section{Palabras clave:}

Filosofía en Chile, prácticas de la filosofía, FONDECYT Regular, ISI, método científico, filosofía como ciencia.

\footnotetext{
(*) Estudiante del Programa de Magíster en Pensamiento Español e Iberoamericano, Universidad Autónoma de Madrid. Espańa. Correo electrónico: matiazsilva@gmail.com

${ }^{1}$ El primer antecedente de esta investigación y la que la precede se remontan a mi tesis de licenciatura de 2008. Una versión acotada y preliminar fue presentada en el I Congreso Nacional de Filosofía, realizado en Santiago de Chile entre los días 6 y 9 octubre de 2009. Quisiera expresar mi sincera gratitud y reconocimiento al filósofo chileno José Santos, sin cuya compañía, apoyo y comentarios, tanto este trabajo como los precedentes serían impensables.

Artículo recibido el 1 de junio de 2012. Aceptado el 4 de marzo de 2013.
} 


\begin{abstract}
This paper tries to analyze the academic practices made by the philosophers that apply for competitive funds. The objective is to show the intentionality in the development of disciplinary researches in order to maintain the exercise of the profession. In this way, the analysis pays attention to one of the guidelines for evaluating used by the competition National Fund for Scientific and Technological Development (FONDECYT) corresponding to the area of philosophy. Thus, it is tried to determine the criterion used to produce and reproduce a specific kind of philosophy following the modern ideal about it: making philosophy as science
\end{abstract}

Keywords:

Chilean philosophy, Practices of philosophy, Regular FONDECYT, ISI, Scientific method, Philosophy as science.

\title{
INTRODUCCIÓN
}

Sin duda la profesionalización de la filosofía en Chile, como remarca Joaquín Barceló, ha representado entre otras cosas, la posibilidad de poder "ganarse la vida” en el ejercicio de la profesión. Como afirma el filósofo chileno, "el carácter público, y en algunos casos oficial, de la organización de su quehacer ha puesto a disposición de la filosofía recursos económicos que hace años habrían sido impensables"2.

La pretensión del escrito que presento a continuación es una aproximación crítica a una de las formas en la que los filósofos chilenos "han sabido ganarse la vida" de manera pública y oficial, como remarca Barceló. Esta práctica de la filosofía chilena a la que intento acercarme es el financiamiento de investigaciones filosóficas por parte del Fondo Nacional de Desarrollo Científico y Tecnológico (FONDECYT) perteneciente a la Comisión Nacional de Investigación Científica y Tecnológica (CONICYT) del Ministerio de Educación.

\footnotetext{
${ }^{2}$ Barceló, Joaquín. "La actividad filosófica en Chile en la segunda mitad del siglo XX". En Fernando Astorquiza (Director). Bio-bibliografía de la filosofía en Chile desde el siglo XVI hasta 1980. Santiago de Chile: Universidad de Chile, Facultad de Filosofía y Educación (1982): 111.
} 
Es pertinente mencionar que este escrito continúa con la línea de investigación que comencé en un trabajo publicado anteriormente en esta misma revista ${ }^{3}$. Por lo mismo, y a manera de introducción, explicitaré algunas ideas que desarrollé en dicho artículo para clarificar el punto de partida y la vocación del presente.

A la luz del concepto de "normalización" filosófica propuesto por el argentino Francisco Romero, que condensa y desarrolla un sentir creciente sobre el avance de la institucionalización de la filosofía latinoamericana ${ }^{4}$, ensayé una revisión de la institucionalización, profesionalización y administración de las prácticas en la filosofía chilena. El enfoque que utilicé, que es en el que se basa también la presente investigación, es el enfoque político-institucional de aproximación a la historia de las ideas. Este enfoque, como señala Cecilia Sánchez, ve a la filosofía conformada también por acontecimientos considerados usualmente externos a esta, sobre todo en lo que respecta a su configuración en un espacio cruzado por algún tipo de poder, concepción que se apoya en la idea de que ningún saber no se agota en sí mismo sino que siempre es administrado de tal o cuál forma ${ }^{5}$. En este ejercicio se mostraron dos cuestiones fundamentales. La primera es que Romero nos presenta una novedosa manera de entender la eterna pregunta ¿qué es filosofía? Desde su visión de la normalización, y en un gesto que me parece muy acertado, esta pregunta puede ser cambiada por esta otra ¿qué prácticas se aceptan como filosóficas? Como es manifiesto, las concepciones "filosóficas" sobre qué es o debiera ser la filosofía alcanza tanta variedad como pudiera imaginarse. Pero a la hora de decidir si un texto es filosofía, las afirmaciones parecen ser más tajantes: un tratado de Zubiri, por ejemplo, si parece serlo, aunque un ensayo de Octavio Paz no. La definición sobre qué es filosofía se mostró como un límite de ciertas prácticas que se aceptan como filosóficas: el estudio y reconocimiento de la sola tradición occidental, una particular disciplina de estudio y escritura, la importancia de becas y distinciones, etc., son algunas de las prácticas que terminan conformando un particular "prestigio filosófico".

\footnotetext{
${ }^{3}$ Para todos los puntos tratados en esta introducción ver: Silva, Matías. "Normalización de la filosofía chilena. Un camino de clausura disciplinar", Universum 24/2 (2009): 172-191.

${ }^{4}$ Este sentir puede vislumbrarse también en el texto de Barceló recién citado, pero con la diferencia de la perspectiva histórica del filósofo chileno (escribe en la década de los 80 a diferencia de Romero que desarrolla su concepto alrededor de los 50) que hace que también pueda vislumbrar aspectos negativos de esta.

${ }^{5}$ Cfr.: Sánchez, Cecilia. Una disciplina de la distancia. Institucionalización universitaria de los estudios filosóficos en Chile. Santiago de Chile: CERC-CESOC, 1992: 82.
} 
La segunda cuestión, tiene que ver con la configuración de un ámbito de validez y administración de dichas prácticas, ámbito que será la disciplina. Con el proceso de institucionalización universitaria en el marco de la modernización de las universidades latinoamericanas, la filosofía empieza a insertarse en el modelo disciplinar de administración del conocimiento. Este modelo está caracterizado por la creciente autonomía de las disciplinas para decidir los métodos y prácticas que la validen como una especialidad. Así, por una fuerte voluntad de esta autonomía, la filosofía chilena se desliga de la dependencia que con respecto del Estado tuvo en los inicios de su inserción institucional universitaria, debido a su rol de apoyo en la formación del profesorado, cuya misión principal era la de formar "ciudadanos" para el naciente Estado de Chile. La Universidad, para la filosofía, será en este sentido "un espacio político-epistemológico cuyo vínculo con el espacio políticoestatal depende del trazado de sus fronteras".

Este ámbito de validez hace que la filosofía, ya disciplinar, se vaya cerrando en criterios intra-académicos de administración de sus prácticas disciplinares, generando un único modelo posible de filosofía que se muestra excluyente tanto de la variedad de fuentes en las que puede circular el pensamiento, de pensadores que no se han circunscrito a la academia y de culturas "filosóficas" completas, por citar los ejemplos desarrollados en el articulo precedente.

El análisis ha mostrado cómo la filosofía administra, a través de una figura particular de "cultura académica", sus límites prácticos que logran decidir qué puede llevar el nombre de filosófico y que no. Pero esto, a mi parecer, debe ser completado con la explicitación de los criterios específicos, el contenido de dicho límite, las características particulares de lo que está siendo tomado, en la práctica, por filosófico. El lugar donde debe rastrearse dicho criterio será en las mismas prácticas que están rigiendo como articuladoras de dicho modelo de filosofía. Este artículo pretende revisar una de aquellas prácticas para ir configurando los matices $y$ alcances de ese criterio que creo necesario dilucidar.

El problema al cuál quisiera acercarme con respecto a este punto dice relación con las formas de evaluación de un proyecto en filosofía para la adjudicación de fondos de investigación. Con esto, pretendo analizar la forma de acceso a uno

${ }^{6}$ Ibíd., 83. 
de los beneficios que la actividad filosófica profesionalmente entendida tiene. Lo importante desde esta perspectiva de análisis será que los criterios que se utilizan para la adjudicación de los fondos nos mostrarán el tipo de filosofía en la que se sostienen y que esta práctica ayuda a reproducir.

El problema se puede poner en los siguientes términos ¿de qué manera se determina qué proyectos financiar y cuáles no? Esta sería la pregunta que la persona o institución que otorgará el financiamiento se hace al momento de llamar a "concurso" para la obtención de fondos.

Se hace necesario entonces determinar un criterio que pueda funcionar de igual forma para todos los postulantes y así poder medirlos bajo un mismo parámetro. Se necesitaría un criterio que ayude a discriminar en base a la conformación de una jerarquía: el proyecto o postulante que esté más cerca del criterio que elijamos será el primero en la lista de beneficiados; por el contrario, los que se quedarán sin el beneficio serán los que queden por debajo de algún mínimo estipulado, o bien, hasta que se agoten los recursos disponibles.

\section{FONDECYT REGULAR: CRITERIOS PARA TODOS LOS PROYECTOS}

En nuestro país, la institución que quizá más prestigio tiene en el financiamiento de proyectos de investigación es la Comisión Nacional de Investigación Científica y Tecnológica (CONICYT). El principal objetivo de la CONICYT es promover, fortalecer y difundir la investigación científica y tecnológica en Chile, para contribuir al desarrollo económico, social y cultural del país ${ }^{7}$. A este organismo pertenece el Fondo Nacional de Desarrollo Científico y Tecnológico (FONDECYT) y dentro de este concurso se encuentra el Programa Regular de Proyectos de Investigación, también llamado FONDECYT Regular, que es el que analizaré en este trabajo ${ }^{8}$. Esta revisión me parece pertinente ya que es uno de los fondos de investigación que más ha aportado fondos para las investigaciones de filosofía por parte del estado de Chile. Para graficar esta importancia puede mencionarse, como ejemplo, que la última etapa de la traducción de Ser y Tiempo

${ }^{7}$ Cfr.: CONICYT_Portal Institucional_Gobierno de Chile- Acerca de CONICYT. Disponible en: <http://www.conicyt. cl/573/propertyvalue_1735.html>. [Consultado: el 4 de febrero de 2008].

${ }^{8}$ Fue creado el año 1982 y apoya proyectos de investigación que tengan una duración de entre 2 y 4 ańos, que cuenten con apoyo institucional y no discrimina por el área o procedencia institucional. Cfr.: Ídem. 
de Eduardo Rivera fue financiada por este fondo, o que Humberto Giannini se lo ha adjudicado en cinco ocasiones.

Los primeros criterios que pueden vislumbrarse en el marco normativo e institucional del FONDECYT Regular son los que la CONICYT tiene por sus "principios claves" para el desarrollo de su misión institucional: excelencia, transparencia y eficiencia? ${ }^{9}$. De estos tres criterios, sólo el primero se refiere a los proyectos que serán apoyados por algunas de las instancias que integran la CONICYT siendo los otros dos, criterios para el correcto funcionamiento de la propia institución. El principio de excelencia significa que los proyectos que apoya la institución deben estar integrados por "investigadores de excelencia” y orientados a lograr "altos estándares de calidad", reflejados en sus "resultados" e "impacto"10. En esta línea, el FONDECYT "financia competitivamente iniciativas presentadas por investigadores con trayectoria demostrada" 11 .

Un segundo lugar en donde se pueden rastrear criterios de evaluación válidos para todos los proyectos que postulan al FONDECYT Regular son las bases de este concurso $^{12}$. En la sección I de "Aspectos Generales" de éstas, en el apartado 1.2 , se establecen las características de todo proyecto que el concurso financie. El texto del 1.2 es el siguiente: FONDECYT financia exclusivamente proyectos de investigación científica o tecnológica, esto es, que conduzcan a nuevos conocimientos o aplicaciones previstas a través de hipótesis de trabajo explicitadas en el proyecto. No financia proyectos de creación artística, recopilaciones, confección de catálogos o inventarios, impresión de libros, ensayos, traducciones, audiovisuales, textos de enseñanza u otras actividades análogas.

Aquí se muestran las dos áreas en que FONDECYT se desenvuelve, la ciencia y la tecnología. Es interesante que el mismo artículo 1.2 especifique qué es lo

\footnotetext{
${ }^{9}$ Cfr.: CONICYT_Portal Institucional_Gobierno de Chile- Misión y Estrategia de CONICYT. Disponible en: < http:// www.conicyt.cl/573/article_7574.html>. [Consultado: el 4 de febrero de 2008].

10 Ídem.

${ }^{11}$ CONICYT_ Portal Institucional_ Gobierno de Chile - Fondecyt. Disponible en: <http://www.conicyt.cl/573/ propertyvalue-1750.html>. [Consultado: el 4 de febrero de 2008].

${ }^{12}$ Las bases que utilizo son las correspondientes a las BASES CONCURSO NACIONAL DE PROYECTOS FONDECYT REGULAR 2008. Disponibles en: <http://www.fondecyt.cl/578/articles-3527_bases.pdf>. [Consultado: el 4 de febrero de 2008], por ser el momento en que comencé esta investigación. Aunque se hayan publicado nuevas bases, no han sufrido mayores cambios en lo que es esencial para esta investigación.
} 
que se entiende por investigación científica y tecnológica. La ciencia, bajo esta descripción, quedaría limitada al rango inespecífico de "conocimiento" pero con una forma específica de producción: su previsibilidad a través de una hipótesis. En este sentido, lo que interesaría al FONDECYT sería la aplicación del "método científico". En la misma presentación de "logros" del FONDECYT se indica que, gracias al financiamiento de este fondo, se ha permitido "que numerosos estudiantes de pregrado, postgrado y profesionales en etapas superiores de formación avancen en su desarrollo intelectual y se familiaricen con el método científico"13. Esto muestra la centralidad que el propio FONDECYT le da a la forma de producción del conocimiento. La tecnología por su parte, se identifica por su "aplicabilidad", manteniendo la misma forma de producción que la de la ciencia.

También es interesante destacar la exclusión de este concurso del "ensayo" y las "traducciones", dos formas en que los filósofos también se han desenvuelto en su quehacer profesional y que han sido desechadas, al parecer, debido a su no adecuación al método científico. Aquí comienza a vislumbrarse lo taxativo de la forma de producción de un conocimiento (sus prácticas), ejemplificado en el método de producción que financiará el FONDECYT Regular.

En el ítem IV, sobre la evaluación de los proyectos, se presentan los "factores de evaluación” con los cuales finalmente se decidirá el financiamiento de los proyectos que estén en la parte superior de la jerarquía que genera toda aplicación de un criterio.

Estos factores son cinco, y se desglosan de la siguiente manera: Calidad de la propuesta 24\%; Relevancia de la Propuesta 12\%; Viabilidad de la propuesta 24\%; Capacidad y productividad del Investigador/a responsable 30\% y Capacidad y productividad de Co-investigadores/as 10\%.

La evaluación de los proyectos consta de dos etapas. En la primera de estas se mide la capacidad y productividad de los Investigadores y de los co-investigadores con una nota en la escala de 1 a 7 . Los proyectos cuyos Investigadores no alcancen una nota en conjunto superior a 3 quedan automáticamente descalificados del

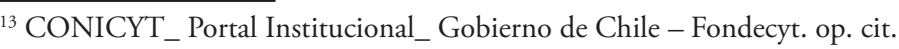


concurso $^{14}$. Con esto se establecería en cierta medida un mínimo de competencia o suficiencia de los investigadores responsables. Podemos pensar que esta competencia asegurará que la investigación que se realice tenga mayores posibilidades de atenerse a los parámetros que tanto la CONICYT como el FONDECYT se han propuesto sobre todo con respecto al principio clave de excelencia. La segunda etapa consiste en la evaluación del proyecto en sí, tomándose en cuenta los otros tres factores involucrados: calidad, relevancia, y viabilidad de la propuesta. A esta etapa sólo acceden los proyectos que no han sido descartados de la etapa anterior y son evaluados por árbitros externos, contactados por el grupo de estudio de la disciplina en cuestión, en nuestro caso, el grupo de estudio de Filosofía ${ }^{15}$.

Cabe destacar las jerarquías de los criterios que el mismo FONDECYT establece. El criterio de Capacidad y productividad de los Investigadores, tomando al responsable y al co-investigador como conjunto, tiene un $40 \%$ de la ponderación final de la evaluación del proyecto siendo el factor con mayor ponderación dentro la evaluación. Luego de esto, y con la misma ponderación se encuentran los factores de Viabilidad y Calidad, con un $24 \%$ cada uno, quedando como factor de menor importancia la Relevancia con un $12 \%$.

Para finalizar con los criterios que valen para todos los proyectos que se presentan

\footnotetext{
${ }^{14}$ Esto sólo desde el 2007.

${ }^{15}$ Vale la pena aquí una explicación de la Organización interna del FONDECYT. La instancia superior del FONDECYT está compuesta por los Consejos Superiores de Ciencia y Desarrollo Tecnológico, los cuales se dividen en el Consejo Superior de Ciencia y el Consejo Superior de Desarrollo Tecnológico. Ambos consejos son independientes entre sí y se relacionan a través de la CONICYT con el gobierno. Los consejos están encargados de la recepción de los proyectos de investigación que se presentan al concurso, fijar las pautas de evaluación de los proyectos, de la selección final de estos y de aprobar o rechazar los Informes Finales. Para estas labores cuentan con la ayuda de los Grupos de Estudio. Los Grupos de Estudio representan el nivel intermedio en la organización del FONDECYT. Estos están encargados de revisar los proyectos postulantes, y en una primera clasificación, descartar los que estuvieren fuera de las bases (contando el criterio de capacidad y productividad). También los Grupos de Estudio están encargados de presentar a los consejos un Informe de Postulación, en donde deben seleccionar los proyectos que, siendo evaluados favorablemente por los árbitros externos, pueden ser financiados con el presupuesto que el consejo correspondiente les ha asignado. Finalmente, se encarga de revisar los informes de avance de los respectivos proyectos y asesoran al consejo pertinente para la aprobación del Informe Final. Los Grupos de Estudio cumplen una labor transversal a la organización del FONDECYT, haciéndose presente en todas las etapas de los procesos de selección y adjudicación de financiamiento por parte del concurso. El sentido que tienen estos dentro de la organización es hacer partícipe a la "comunidad científica" nacional dentro de los procesos tanto en la evaluación de los proyectos como en la colaboración con los Consejos Superiores. Es por esto que los Grupos de Estudio están en constante renovación de sus integrantes. FONDECYT tiene 25 grupos de estudios, los cuales representan a 19 disciplinas o ámbitos del saber (Biología, Medicina e Ingeniería cuentan con 3 grupos de estudios cada uno). Uno de estos grupos de estudios es el de Filosofía. Como todos los demás está conformado por un Director de grupo, un Consejero, un Coordinador de Proyectos y una cantidad de miembros dependiendo del grupo de estudio (3 en el Grupo de estudio del FONDECYT Regular 2008). Cfr.: FONDECYT -Fondo Nacional de Desarrollo Científico y Tecnológico- Acerca de FONDECYT. Disponible en: <http://www.fondecyt.cl/578/propertyvalue-57377.html>. [consultado: el 8 de Febrero de 2008].
} 
al FONDECYT Regular quiero reparar en el ítem III de las bases "Derechos y Obligaciones". Las bases establecen, en su apartado 3.7, los requisitos para dar por terminado un proyecto de investigación. El proyecto se da por concluido cuando se ha aprobado el Informe Final que debe presentar el Investigador responsable al Consejo Superior de Ciencia y de Desarrollo Tecnológico atingente al mismo, siendo aceptada, además, la declaración de gastos del proyecto. Dos son los requisitos que se piden para que el Informe Final sea aprobado: que se hayan cumplido los objetivos propuestos por la investigación y que se hayan cumplido con los requisitos "mínimos" en cuanto a publicaciones. Estos requisitos valen para todos los proyectos, no importando el área de la investigación, y son los siguientes: un manuscrito aceptado en una revista incluida en la base de datos ISI o equivalente para los proyectos de dos y tres ańos, y dos manuscritos de iguales características para los proyectos de cuatro años.

En este punto me gustaría destacar dos cosas. La primera, es que los únicos requisitos "externos" con respecto a las investigaciones tienen relación con que sus resultados tienen que ser publicados. La segunda se refiere al patrón de esas publicaciones: tienen que presentarse manuscritos aprobados por publicaciones que pertenezcan a "la bases de datos ISI" o sean similares a esta.

Finalmente, dentro del marco institucional y normativo en el que se inserta el FONDECYT Regular y que funcionan para los proyectos en todas las áreas, podemos extraer los siguientes criterios de evaluación de proyectos:

En primer lugar tenemos un grupo de criterios que están orientados a la evaluación de los investigadores. De estos se pide que sean "investigadores de excelencia” con "trayectoria demostrada”. Estas dos características se medirían, según lo expuesto, bajo los parámetros de su capacidad y productividad.

El segundo grupo de criterios que podemos encontrar en este marco general tiene que ver con los proyectos mismos. De estos se pide "altos estándares de calidad" que pueden medirse en sus "resultados" e "impacto". La calidad de los proyectos, paradójicamente, tendrá un segundo plano de relevancia con respecto a los criterios utilizados para con los investigadores, y se medirá directamente en los proyectos por árbitros externos. Por otro lado los resultados y el impacto se 
medirán de dos formas: de manera directa en el proyecto con el cumplimiento de los objetivos propuestos del proyecto, y por la exigencia de la aceptación de uno o dos manuscritos en revistas que pertenezcan al catálogo ISI, o que sean equivalentes a estas.

Un tercer aspecto importante es que se pide un método específico de producción de conocimiento, el método científico, que permite que los conocimientos a los que se llega sean "previsibles". Finalmente, con la menor importancia, se ubica el criterio de la relevancia del proyecto.

Hasta aquí sólo he rescatado nítidamente un solo criterio en cuanto a la forma de producción de conocimientos dentro de los cuales participará la filosofía: el modelo de filosofía que se financie deberá atenerse al método científico, en su forma más amplia de la predictibilidad de resultados a través de una hipótesis, como forma de producción de su saber. Pero falta aún desentrañar dos puntos centrales. El primero de estos es qué significa o cómo se mide la "capacidad" y "productividad" de un investigador. En otras palabras, cómo FONDECYT se asegura de la "suficiencia investigativa" de éste. El segundo punto tiene que ver con la medición de la calidad del proyecto. Aquí lo que falta es desentrañar el criterio que el FONDECYT coloca como paradigma para medir la calidad de una investigación en sus resultados: la base de datos ISI. Revisaré entonces los criterios que la base de datos ISI utiliza para aceptar o rechazar a revistas dentro de su catálogo.

\section{CRITERIOS PARA INVESTIGACIONES EN FILOSOFÍA}

Para poder desentrańar estos dos aspectos que quedan, y que, como mostraré, están estrechamente relacionados, me enfocaré primero en el ámbito específico en el que compiten los proyectos de Filosofía. Este ámbito está normado por una "Pauta de evaluación curricular" para evaluar la "capacidad" y "productividad" de los postulantes y que vale de manera general para el área de Ciencias Sociales y Humanidades a la que pertenece el Grupo de Estudio de Filosofía (Figura I). Esta pauta es el lugar de máximo acercamiento del FONDECYT con la filosofía, el lugar de mayor especificidad previa a la evaluación de los árbitros externos a FONDECYT. 
Algunas cosas son importantes de destacar de la pauta. El criterio de "capacidad" de un investigador, que es un 40\% del total de la evaluación de la "Pauta", se mide bajo dos parámetros: con respecto a la carrera académica, que haya desarrollado el investigador o que esté ayudando a desarrollar (exclusivamente en dirección de tesis), y por las investigaciones que se haya adjudicado. Estas investigaciones deben seguir el mismo modelo que las que propone FONDECYT o uno de los organismos "internacionales" con los que tiene convenio.

Del ítem de "productividad", que representa el otro 60\%, cabe destacar dos cuestiones de carácter general. La primera es el evidente (pero siempre a-crítico) monopolio de la palabra escrita por sobre otras formas de expresión del pensamiento, ya que el único indicador de productividad para el FONDECYT Regular es el de "publicaciones". La segunda es que, para la productividad se miden sólo los últimos cinco años de producción, lo que estaría asegurando que el investigador esté en plena "etapa productiva”.

Por último, parece más que sugerente que el Proyecto SciELO, creado como una instancia regional latinoamericana para la difusión de los resultados de la información científica, atendiendo a modelos como el ISI, pero buscando adecuarse a las características regionales en cuanto a la publicación y difusión de la información científica, tenga un menor puntaje que el criterio ISI generado por una empresa privada. Sugerente aún más es que SciELO además esté conformado por organismos públicos e internacionales de desarrollo científico; y que sea un proyecto del cual CONICYT es parte integrante ${ }^{16}$.

La pregunta que surge de inmediato, y que tendré que intentar responder, es ¿por qué el criterio ISI? En cierta manera, al medir la calidad de la productividad bajo este criterio, lo que hace FONDECYT es "externalizar un servicio". No utiliza un criterio interno ni autoimpuesto de evaluación de calidad, sino que recurre a uno externo. ¿Por qué?

${ }^{16}$ Risible es que la misma sigla de este proyecto nacido en Brasil, y proyectado para una comunidad hispano y luso-parlante este en inglés: Scientific Electronic Library Online. 


\section{EL CRITERIO ISI DE PUBLICACIÓN}

ISI es la sigla que designa al Insitute for Scientific Information, nombre que tenía antes la actual compañía Thomson Scientific ${ }^{17}$, una empresa privada de Estados Unidos que publica bases de datos de revistas científicas, y que tiene por misión principal "proporcionar amplia cobertura a las investigaciones más importantes e influyentes realizadas en todo el mundo"18. Esta empresa se preocupa principalmente de agregar revistas de publicaciones científicas de todas las disciplinas a sus catálogos. Una revista que sea agregada a la base de datos del ISI tiene que cumplir, sin embargo, ciertos requisitos específicos, que asegurarían que aquellas revistas publican conocimientos científicos de relevancia y calidad.

Thomson Scientific cubre en sus bases de datos la información sobre revistas especializadas en ciencia, ciencias sociales, artes y humanidades. La compañía, en cuanto al proceso de evaluación de revistas, está constituida por un equipo editorial integrado por personas que "cuentan con una formación educacional apropiada para sus áreas de responsabilidad, así como experiencia e instrucción en la ciencia de la información" ${ }^{19}$. El objetivo del equipo editorial es principalmente identificar y evaluar las revistas nuevas y prometedoras que pueden ser de interés para las personas que utilizan la base de datos ISI, y también excluir de la base las revistas que han dejado de ser pertinentes o menos útiles. Thomson Scientific pretende tener en su base de datos lo que llaman un "núcleo esencial de revistas" que alcanzan actualmente alrededor del 10 o 12\% de los títulos evaluados (alrededor de 2000 al ańo $)^{20}$.

Para la evaluación de las revistas especializadas, ISI utiliza una serie de criterios cuantitativos y cualitativos que son interrelacionados para poder finalmente aceptarla dentro de la base de datos. Lo primero que se evalúa son una serie de

\footnotetext{
${ }^{17}$ En adelante ISI, por ser el nombre más reconocido.

${ }^{18}$ Testa, James. "La base de datos del ISI y su proceso de selección de revistas", ACIMED [online] 9/4 (2001): 138-140. Disponible en: <http://scielo.sld.cu/scielo.php?script=sci_arttext\&pid=S1024-94352001000400023\&lng=es\&nrm=iso >. [consultado: el 4 de Febrero de 2008] James Testa era Gerente del Departamento Editorial del Institut for Scientific Information, al momento de la presentación de este artículo en el Seminario sobre Evaluación de la Producción Científica, realizado en São Paulo por el Proyecto SciELO, del 4 al 6 de marzo de 1998.

${ }^{19}$ Ídem.

${ }^{20}$ En los años '50 C.S. Bradford percibió que la mayor cantidad de los trabajos científicos eran publicados en un número muy reducido de revistas, que constituyen el núcleo de las publicaciones de una disciplina particular. Como este núcleo no es estático, la revisión de Thomson de la literatura científica se actualiza cada dos semanas. Cfr.: Ídem.
} 
patrones básicos para las revistas. El primero de estos patrones es la periodicidad regular de la publicación. Otro criterio utilizado es que las revistas cumplan con los requisitos editoriales internacionales. Estos requisitos son, entre otros, títulos informativos de las revistas, títulos de artículos y resúmenes completamente descriptivos, datos bibliográficos completos en todas las referencias y la dirección de cada autor. También es esencial que, tanto los títulos, resúmenes y palabras claves estén en inglés, esto, aduciendo el mayor número de lectores posibles. Finalmente, se pide que las revistas apliquen el sistema de revisión por pares, esto es, a través de árbitros o referees que evalúen los artículos que se aceptan en las revistas. Esta revisión se pide para poder asegurar, de cierta manera, la calidad de las investigaciones que son publicadas además de la corrección de las referencias que los artículos citan ${ }^{21}$.

Otros tres criterios son importantes para Thomson Scientific: el contenido editorial, la internacionalidad, y el análisis de citas. El primero de estos tiene relación con la emergencia de nuevos temas de interés dentro del desarrollo de las disciplinas. Los editores ISI se preocupan de ver si la línea editorial de la revista en cuestión se refiere a temas novedosos o por el contrario el tema ya está "cubierto" por otras revistas que compongan la base de datos. El criterio de internacionalidad por su parte se relaciona con la necesidad de satisfacer a un público amplio, para lo cual se intenta agregar a la base de datos revistas en donde, tanto los autores que aparecen en la revista como los autores de los artículos citados, sean de diversos lugares y nacionalidades.

El último de estos criterios, y que James Testa, ex-director del Departamento Editorial del ISI, destaca como de los más importantes para la evaluación de revistas, es el análisis de citas. Para esto ISI edita una publicación anual llamada Journal Citation Reports que tiene dos ediciones: la Science Edition y la Social Sciencie Edition. Como se afirma en la página institucional de Thomson Scientific, este instrumento "ofrece un medio objetivo y sistemático para evaluar las revistas más importantes del mundo. Ofrece una perspectiva única para la evaluación y la comparación de revistas, ya que acumula y tabula el número de citas y artículos de prácticamente todas las especialidades de las ciencias, la tecnología y las ciencias sociales"22.

\footnotetext{
${ }^{21}$ Cfr.: Ídem.

${ }^{22}$ Tarjeta de consulta rápida de Journal Citation Reports. Disponible en: <http://scientific.thomson.com/media/scpdf/jcr1005-q-sp.pdf>. [consultado: el 8 de Febrero de 2008].
} 
Recordemos que el criterio de "productividad" dentro de la pauta de evaluación curricular de FONDECYT que corresponde a Filosofía, está regido por el paradigma de las normas ISI para la inclusión de revistas en sus bases de datos. El factor de productividad se evalúa en el investigador tanto para adjudicar o rechazar los fondos a un proyecto de investigación, como en la investigación misma, para poder darla por concluida.

Las características principales que el criterio ISI de evaluación comporta, tienen que ver con el acceso a información, tanto cualitativa como cuantitativa, sobre las publicaciones que están es su base de datos. Con esto no califican directamente la calidad de una revista o artículo con criterios estáticos definidos heterónomamente con respecto a una disciplina en particular, sino que, la calidad se califica por el nivel de aceptación, difusión e impacto que la revista tiene en la llamada "comunidad científica", por eso la revisión por pares se les exige a cada revista como sistema de evaluación. Lo realmente evaluado no es el contenido de los artículos (esto no podría ser convertido en algún factor “objetivo") sino el nivel de citación que tiene en otras revistas, que han de estar por supuesto, incluidas en las bases de datos ISI. Del criterio ISI creo que podemos rescatar dos cosas esenciales. La primera de estas tiene que ver con el sistema de "revisión por pares”. Este sistema de evaluación, así como la aparición de las revistas científicas como medio institucionalizado, nacen y se afinan al alero del desarrollo de la producción científica en los países de Europa central. Tiene como antecedentes más remotos la aparición de las dos primeras revistas de publicación científica, la Journal des Savants en Francia y la Philosophical Transactions, dependiente de la Royal Society of London for Improving Natural Knowledge, ambas en 1665. En esta última se hace explícito que la revista debe ser revisada por algún miembro de la Royal Society antes de su publicación. Esta presentaba las características institucionales primitivas de las revistas científicas actuales: tener un director, una estructura editorial, una institución responsable, edición periódica, revisión por pares y reconocimiento de los autores ${ }^{23}$. La "ventaja" de este criterio, que utiliza FONDECYT para evaluar sus proyectos, es que la evaluación de la calidad se hace en un reducto de autonomía disciplinar de manera autónoma, a través de la aceptación por parte de los pertenecientes al ámbito disciplinar en cuestión y no

\footnotetext{
${ }^{23}$ Cfr.: Camps, Diego. "El artículo científico: Desde el inicio de la escritura hasta la IMRYD”, Archivo de Medicina [online] 3/5 (2007): 3-4. Disponible en: <http://archivosdemedicina.com/ojs/index.php/archmed/article/view/57/97>. [consultado: el 24 de Febrero de 2008].
} 
estableciéndose criterios externos a la disciplina para la toma de decisiones. Por esto se hace muy importante dentro de la disciplina el "hacerse de un nombre", tener una reputación, un prestigio. Y por esto es que el sistema de "citas" ha llegado a semejante nivel de complejidad. Podemos saber hoy, por ejemplo, cuál es el científico chileno, por área de especialidad, que tiene mayor número de citas a nivel mundial ${ }^{24}$.

Es notorio que este criterio cristaliza la institucionalidad que se venía sucediendo junto al mismo desarrollo de la ciencia, esto es, el modelo disciplinar del conocimiento. A través de la observación del sistema de revisión por pares, por ejemplo midiendo la cantidad de citaciones que tienen los referees y los miembros del comité editorial dentro de las publicaciones de la base de datos ISI (lo que le daría un peso y un prestigio a la publicación), se estaría dejando el criterio de validación a la autonomía disciplinar, a las prácticas y criterios propios de cada una de las áreas del saber. Asumiendo para la filosofía chilena el marco en el que he situado esta investigación, esto significaría que el criterio finalmente recaería en los que administran las formas de producción filosófica: la "cultura académica".

El segundo aspecto a destacar está íntimamente relacionado con el anterior y tiene que ver con una suerte de criterios mínimos con respecto a la producción de los textos que han de ser publicados en las revistas que pueden entrar dentro de la base de datos y que corresponden a los requisitos editoriales internacionales ya mencionados. Estos criterios tienen que ver tanto con una mayor amplitud de lectores posibles como con respecto a la fácil administración de los datos de cada artículo. El problema central aquí, que da razón de la existencia de una empresa como Thomson Scientific es, como lo enuncia Diego Camps, que el aumento sustancial de las publicaciones científicas hace que no sólo sea necesario el acceso a la información, sino que se hace indispensable adquirir una destreza para seleccionar la información relevante ${ }^{25}$. En este sentido es Thomson Scientific el que hace el trabajo por nosotros, y como se ve, también hace el trabajo por FONDECYT.

\footnotetext{
${ }^{24}$ Destaca aquí una de las herramientas creada por Thomson y que es el llamado "factor de impacto". A través de esta, puede conocerse el nivel de citaciones que, tanto autores como revistas, tienen dentro de la misma base de datos ISI. Cfr.: Tarjeta de consulta rápida de Journal Citation Reports, op. cit.

${ }^{25}$ Camps, Diego. "El artículo científico", op. cit., 5.
} 
La respuesta a la interrogante sobre por qué ocupar el criterio ISI para la evaluación de la productividad de los investigadores y sus investigaciones por parte de FONDECYT se hace evidente y la explica Anna Prat, Jefa del Departamento de Información de la CONICYT en 1998: “(p)ara crear nuestros indicadores y hablar de productividad científica, utilizamos únicamente el ISI, porque si queremos comparar datos y medir nuestra ciencia con la ciencia internacional, debemos usar a una medida común a todos" 26 .

Por esto hablo de criterios "mínimos" de producción, porque los criterios mínimos son exigidos a todos, con el fin de poder realizar comparaciones entre las distintas revistas y así poder "seleccionar" aquellas de mayor relevancia. En definitiva, los criterios mínimos están puestos ahí por un problema de administración de la producción científica. Se pretende ampliar, de esta forma, la "validez" de las investigaciones: los criterios que se utilizan están ahí porque se creen los de mayor "neutralidad" o por lo menos "eficiencia" con respecto a la transmisión de los resultados de las investigaciones. Lo que se intenta, en definitiva, es llegar a una suerte de criterios "objetivos" con respecto a publicación de conocimientos.

La "objetividad" alcanzada por este tipo de parámetros, ya no remite sin embargo a una universalidad que pueda o quiera valer "para todo ser racional", sino que es una que está valiendo de facto para las distintas "comunidades científicas" en el mundo, para las distintas disciplinas. Tanto el criterio de revisión por pares, como el de los requisitos mínimos de producción de un texto estarían asegurando ese tipo de objetividad o validez.

\section{EL CRITERIO Y SU FUNCIONAMIENTO}

Falta responder entonces a dos interrogantes: ¿cuál es la idea de filosofía que se desprende de las prácticas analizadas? ¿cómo se inserta en este tipo de sistema de administración del saber?

Frente a la primera pregunta, que tiene que ver con el contenido del límite

\footnotetext{
${ }^{26}$ Prat, Anna. "Evaluación de la producción científica como instrumento para el desarrollo de la ciencia y la tecnología", ACIMED [online] 9/4 (2001): 111-114. Disponible en: <http://scielo.sld.cu/scielo.php?script=sci_arttext\&pid=S102494352001000400016\&lng=es\&nrm=iso >. [consultado: el 5 de Febrero de 2008] Jefa del Departamento de Información. Comisión Nacional de Investigación Científica y Tecnológica (CONICYT), Chile.
} 
disciplinar de la filosofía dado por esta práctica, hemos dilucidado los siguientes criterios de evaluación de proyectos: primero, la investigación debe seguir como criterio de producción de su conocimiento la observación del método científico, esto es la previsibilidad de su conocimiento a través de una hipótesis. Segundo, el investigador debe estar debidamente "capacitado" para este tipo de investigaciones, esto es, debe tener una carrera académica de alto nivel (ojalá Doctor) y estar ayudando a reproducir dicha carrera. Además, debe preferentemente haber ganado algún concurso anterior de este tipo, teniendo como parámetro los mismos concursos financiados por la CONICYT o institutos con los que tiene convenios. Y tercero, debe tener un alto nivel de "productividad" esto es, debe haber publicado su "producción" intelectual en revistas "reconocidas" en donde el mayor grado de reconocimiento es para las revistas que se incluyen en el catálogo ISI. Este catálogo tiene como principales criterios el que las revistas utilicen el sistema de revisión por pares y un "mínimo" de normas de producción de un texto que impliquen un fácil manejo de la información que contengan. Estos criterios, huelga decirlo, fueron desarrollados para hacer frente a las necesidades que la ciencia fue generando en el desarrollo de su saber.

De estos criterios podemos distinguir entre unos que funcionan como paradigma y otros que funcionan como reproductores de dicho paradigma. El paradigma está establecido tanto por la aplicación del método científico, como por el sistema actual de producción, administración y difusión del saber científico, en donde el criterio ISI y la carrera académica serían los principales filtros de evaluación y por ende las principales características del tipo de filosofía producida y promovida por FONDECYT. Con esto, podemos deducir que, en cierta medida, la aspiración teórica de muchos proyectos filosóficos como los de Kant y Husserl, sería la aspiración que la filosofía disciplinar chilena tiene en la constitución práctica de sus límites, visto exclusivamente desde los criterios que esta práctica ayuda a producir y reproducir: hacer filosofía como ciencia.

Desde un primer momento la exigencia de que las investigaciones financiadas produzcan sus conocimientos bajo la amplia pero igualmente estricta forma del método científico se pone como criterio fundamental. Podría aducirse que en realidad es una parte muy pequeña de la producción filosófica chilena la que se somete a este criterio. Pero más allá de que el criterio tenga un reducido espacio 
de aplicación (que me parece reducido más bien por falta de fondos que por otro motivo), lo importante es que comporta una de las maneras en que la filosofía se ha ido institucionalizando y profesionalizando.

En segundo lugar, tenemos que ver que existen dos paradigmas con respecto a la evaluación de los proyectos: el criterio ISI y la evaluación por pares (que ISI también recoge como un criterio propio) Estos dos criterios han surgido y desarrollado al alero de la ciencia moderna y como respuesta a sus problemas de producción, administración y difusión de su saber.

Con respecto a la evaluación por pares, el punto crucial se encuentra en la voluntad de "autonomía" en la institucionalización disciplinar de la filosofía chilena. Como revisé en el artículo que precede a este, la filosofía se constituye como disciplina en una doble necesidad de reconocimiento. Por un lado el reconocimiento social; por otro, el reconocimiento al interior de la universidad. Para poder ganarse un espacio dentro de la universidad los filósofos chilenos utilizan dos estrategias: la de administrar autónomamente sus límites disciplinares y la de erguirse como una disciplina que ocupa ciertas técnicas específicas en la constitución de su saber. La búsqueda de reconocimiento en el espacio universitario conlleva un cierto "costo" que muestra una toma de postura con respecto a la definición práctica de la filosofía y que funciona tras la inserción de la filosofía en este sistema de administración del conocimiento.

La filosofía entra a "disputar" un lugar de reconocimiento dentro de un modelo nuevo de universidad. La universidad chilena y latinoamericana, alrededor de los años 50, entra en un proceso de modernización. Como mencioné, esta modernización toca dos aspectos que me interesa rescatar aquí. El primero de estos es que la vocación de esta modernización es asegurar la autonomía de cada disciplina, la articulación del saber dentro de la universidad como una serie de disciplinas. Por este motivo la filosofía entraría en el proceso de definición de sus fronteras en coordenadas intra-académicas. Pero este mismo proceso de modernización lleva a la universidad a plantear la adecuación de las enseñanzas al desarrollo de la ciencia y la tecnología a nivel internacional, esto es, el modelo disciplinar sumado a la alianza entre conocimiento y producción ${ }^{27}$.

\footnotetext{
${ }^{27}$ Cfr.: Silva, Matías. "Normalización de la filosofía chilena”. op. cit.
} 
Aunque creo correcta la estimación de Cecilia Sánchez, según la cual el nivel de compromiso de la filosofía chilena con este esquema del saber que impulsa la modernización de la universidad es bajo (por la auto-marginación de la nueva alianza entre conocimiento y producción $)^{28}$ creo que este alcanza a permear las prácticas filosóficas en la medida en que se asume ese nuevo modelo institucional de universidad. Esta permeabilidad se da a través de la importación de una forma de hacer filosofía, que viene aparejada al modelo universitario europeo. La filosofía disciplinar chilena, si bien se desentiende de la "productividad" de su saber, no logra desentenderse de las "formas de producción" que el esquema disciplinar trae consigo debido a un problema de administración del saber. Así la filosofía, al contrario de la intención de la misma postura "autonómica" de su constitución disciplinar, no logra ser aséptica a los contextos sociales en que se desarrolla.

Éstas formas de producción del saber, que hemos calificado como ciertos "mínimos" exigidos a todos los proyectos e investigadores evaluados, son los que rigen en el criterio ISI de publicación y muestran un segundo aspecto en que la filosofía estaría, en la asunción de este modelo de administración del saber, intentando, en la práctica, ser una ciencia. No sólo es porque este sistema haya nacido a la par que la ciencia, sino porque se basa en dos aspectos que en este trabajo quisiera dejar sólo anotados y que tocan directamente a la constitución moderna de la ciencia: el manejo de la información reducida a ciertos parámetros cuantificables y la pretensión de universalidad de estos criterios.

En el primero de estos resuena la idea de Galileo, uno de los precursores de la ciencia moderna, de que la realidad está escrita en lenguaje matemático y que sólo basta aprender a leerlo. Esta afirmación, en lo que nos interesa aquí, estaría indicando que la realidad puede ser cuantificable, equiparable y, por ende, manejable. En este sentido es ilustrativo que, por el problema de acceso y organización que apunta Diego Camps, se hable de "ciencias de la información" pensando que el círculo del desarrollo de la investigación científica no se cierra con los resultados de una investigación sino que con su "correcta” y "amplia” difusión ${ }^{29}$.

Con respecto a la segunda cuestión, lo que ocurre en la inserción de la filosofía en el

${ }^{28}$ Cfr.: Sánchez, Cecilia. Una disciplina de la distancia. op. cit., 92-93.

${ }^{29}$ Cfr.: Prat, Anna María. "Evaluación de la producción científica...”. op. cit. 
sistema actual de administración de la producción del saber en occidente es lo mismo que ocurre en la inserción de la filosofía en el contexto universitario: si el primer sistema hace "objetiva" la validez de unos criterios debido a que estos funcionan de facto para las diversas comunidades científicas, la modernización de la universidad institucionaliza el modelo de enseñanza como una adecuación a estas pautas del desarrollo científico y tecnológico, sistema que es válido en la práctica actualmente.

La universalidad en este sentido, que se resuelve en una validez objetiva y neutra, se gana no en cuanto a una validez teórica de los postulados (como la universalidad de la metafísica moderna) sino que a una validez de facto de sus prácticas. En esto se juega, por ejemplo, la importancia del método tanto para la ciencia moderna como para las prácticas filosóficas: es una universalización práctica, que no necesita ser válida para todo ser racional, sino que de facto está valiendo para el desarrollo de la ciencia a nivel mundial ${ }^{30}$.

¿De dónde saca su fuerza este criterio de la universalidad práctica? Dicho de otra forma ipor qué la filosofía se constituye como filosofía disciplinar, poniendo un criterio rígido entre lo que es y no es filosofía, hipotecando en alguna medida su voluntad de autonomía disciplinar? El problema principal de los criterios que esta práctica reproduce es el mismo que encontrábamos en la institucionalización de la filosofía chilena como filosofía disciplinar: la instalación de un modelo único de filosofía, filosofía como ciencia desde el análisis de esta práctica, que excluye a muchas otras formas de filosofía posible. A esta "actitud" la hemos querido llamar "clausura disciplinar". Podría replicarse que este puede ser un modelo de filosofía como muchos otros, pero aquí el punto principal es insistir en que la filosofía, el pensamiento, no es aséptico a sus condiciones de producción sino que está cruzado y constituido por ellas; en el caso de la filosofía chilena, que existe una administración rígida de sus límites disciplinares.

Para graficar cómo funciona esta reproducción de un modelo "único y excluyente" de filosofía es suficiente con mostrar la importancia institucional que tiene este modelo de producción de filosofía, que se vuelve clave a la hora de "vivir de la filosofía”. Anna Prat, comenta:

\footnotetext{
${ }^{30}$ Queda pendiente el desarrollo de estos puntos en un trabajo posterior, ya que su desarrollo implicaría un trabajo que sería por completo autónomo de este.
} 
"En una ocasión, un rector me contaba que el ministerio le había informado que a su universidad le faltaba un artículo y ese artículo, publicado aunque no contabilizado, significaba un profesor a tiempo completo por año [...] Lamentablemente, las universidades han comenzado a utilizar estudios de impacto, número de citas, número de artículos en revistas corrientes principales, etc., para promover la carrera académica"31.

Me pregunto si serán muy distintos los criterios que utilizarán los Departamentos de Filosofía de las universidades cuando llaman a concurso para contratar a nuevos Académicos, sobre todo si un profesor que tenga publicaciones ISI puede revestir tal importancia económica para la institución. En la práctica, este "modelo" de filosofía que se encuentra muy arraigado ya en el desarrollo profesional de la filosofía en Chile, se caracteriza por una creciente homogeneización. La clausura extrae su fuerza e importancia de la misma posibilidad de ganarse la vida haciendo filosofía.

Recordemos que, desde nuestra perspectiva de análisis, son sus prácticas las que definen a la filosofía y, en este sentido, los criterios que se utilizan en el concurso FONDECYT Regular están funcionando como criterio para la distinción de la auténtica filosofía. En la práctica profesional el criterio ISI, por ejemplo, nos sirve para "identificar" la información relevante sin siquiera haber leído nada: si una reflexión no es generada o presentada en la forma académicamente aceptada, simplemente puede dejarse de lado, sin el remordimiento de haber podido perder algo valioso para la filosofía disciplinariamente entendida. En pos de una mejor administración de los "recursos bibliográficos" y del tiempo de investigación, se da también una clausura de las "fuentes" desde donde se trabaja la filosofía.

La aceptación de este criterio de evaluación y, en definitiva, de este modelo de filosofía, tiene costos para la filosofía relacionados con esta exclusión que, a mi parecer, son altísimos si empezamos a pensar seriamente en qué filosofía chilena queremos. Sobre todo si pensamos que este sistema es un sistema que se autoreproduce como modelo, no sólo en la práctica profesional propiamente dicha, sino que también en la enseńanza y el estudio de la disciplina al interior de los departamentos y facultades de filosofía, prácticas de enseñanza y estudio que será necesario analizar en trabajos posteriores.

${ }^{31}$ Ídem. 
No pretendo, con el análisis de esta sola práctica, afirmar que la filosofía disciplinar pretende ser una ciencia en su totalidad, no sólo porque haga falta analizar de manera más exhaustiva otras prácticas disciplinares, sino porque creo que dentro de la filosofía disciplinar concurren diversas vertientes o tradiciones de prácticas de pensamiento en donde existe la posibilidad de disenso y conflicto. Esto no niega sin embargo que existan prácticas mejor y peor valoradas dentro de la academia: en una metáfora espacial, algunas se sitúan más cerca de lo que podría ser el "centro"; otras hacia la periferia. Por esto he querido llamar al lugar o forma de administración de dichas prácticas una cierta "cultura académica". De esta cultura puede derivarse una actitud de "academicismo" 32 , como una administración rígida de ciertas prácticas, pero este academicismo no lo identifico sin más como la única manera de permanecer dentro de los límites disciplinares: hacer filosofía como ciencia no es la única manera de hacer filosofía disciplinariamente entendida, aunque si sería actual y prácticamente, la manera que tiende a producirse y reproducirse.

\section{REFERENCIAS}

Barceló, Joaquín. "La actividad filosófica en Chile en la segunda mitad del siglo XX”. En Fernando Astorquiza (Director). Bio-bibliografía de la filosofía en Chile desde el siglo XVI hasta 1980. Santiago de Chile: Universidad de Chile, Facultad de Filosofía y Educación (1982): 111.

Bases concurso nacional de proyectos FONDECYT Regular 2008. Disponible en: <http: //www.fondecyt.cl/578/articles-3527_bases.pdf.>. [consultado: el 4 de Febrero de 2008].

Camps, Diego. "El artículo científico: Desde el inicio de la escritura hasta la IMRYD”, Archivo de Medicina [online] 3/5 (2007). Disponible en:<http:// archivosdemedicina.com/ojs/index.php/archmed/article/view/57/97>. [Consultado: el 24 de Febrero 2008].

\footnotetext{
$\overline{{ }^{2} 2 \text { Una especie de etnocentrismo de esta cultura. }}$
} 
Concurso FONDECYT Regular 2008 - Pauta de evaluación curricular Ciencias Sociales y Humanidades. Disponible en: <http://www.fondecyt.cl/578/ article-27531.html>. [Consultado: el 8 de Febrero de 2008].

CONICYT_Portal Institucional_ Gobierno de Chile. Disponible en: <http:// www.conicyt.cl>. [Consultado: el 1 de Febrero de 2008].

FONDECYT- Fondo Nacional de Desarrollo Científico y Tecnológico. Disponible en: <http://www.fondecyt.cl>. [Consultado: el 16 de Diciembre de 2007].

Prat, Anna María. "Evaluación de la producción científica como instrumento para el desarrollo de la ciencia y la tecnología", $A C I M E D$ [online] mayo 2001, 9/4 (2001): 111-114. Disponible en: <http://scielo.sld.cu/scielo. php?script=sci_arttext\&pid=S1024-94352001000400016\&lng=es\&nrm= iso>. [Consultado: el 26 Febrero 2008].

Sánchez, Cecilia. Una disciplina de la distancia. Institucionalización universitaria de los estudios filosóficos en Chile. Santiago de Chile: CERC-CESOC, 1992.

-----a---. "Formas de circulación institucionales de la filosofía en Latinoamérica", Universum 11 (1996): 189-200.

Silva, Matías. "Normalización de la filosofía chilena. Un camino de clausura disciplinar”, Universum 24/2 (2009): 172-191.

Tarjeta de consulta rápida de Journal Citation Reports. Disponible en: <http:// scientific.thomson.com/media/scpdf/jcr-1005-q-sp.pdf>. [Consultado: el 6 de Febrero de 2008].

Testa, James. "La base de datos del ISI y su proceso de selección de revistas", $A C I M E D$ [online] mayo 9/4 (2001): 138-140. Disponible en: <http://scielo.sld.cu/ scielo.php?script=sci_arttext\&pid=S1024-94352001000400023\&lng=es \&nrm=iso>. [Consultado: el 4 Febrero 2008]. 
Figura $I^{33}$

\section{Concurso FONDECYT Regular 2008 \\ Pauta de Evaluación Curricular \\ Cs. Sociales y Humanidades}

\begin{tabular}{|c|c|c|c|}
\hline 1.- Capacidad $(40 \%)$ & Puntos & Frc. IR & Puntaje IR \\
\hline \multicolumn{4}{|l|}{ 1.1. FORMACIÓN (no se suma, se considera el grado más alto obtenido) } \\
\hline Doctorado & 10 & & 0 \\
\hline Magister, Master, D.E.A & 5 & & 0 \\
\hline \multirow[t]{2}{*}{ Titulo Universitario o Licenciatura } & 3 & & 0 \\
\hline & ( máximo 10) & Total & 0 \\
\hline \multicolumn{4}{|c|}{ 1.2. INVESTIGACIONES CONCURSADAS Y FINACIADAS (Como coinv, mitad de puntaje) } \\
\hline FONDECYT, FONDEF, Milenio, Anillos, NSF, CNRS ( Max. 10 puntos) & 5 & & 0 \\
\hline \multirow[t]{2}{*}{$\begin{array}{l}\text { Proyectos competitivos financiados por Deptos. de Investigación, Universidades e } \\
\text { Instituciones y Centros de Investigación (Max. } 6 \text { puntos) }\end{array}$} & 2 & & 0 \\
\hline & ( máximo 16) & Total & 0 \\
\hline \multicolumn{4}{|l|}{ 1.3. DIRECCIÓN DE TESIS de Magíster y Doctorado } \\
\hline Guia de tesis de Doctorado terminadas y aprobadas & 2 & & 0 \\
\hline \multirow[t]{2}{*}{ Guia de tesis de Magister terminadas y aprobadas } & 1 & & 0 \\
\hline & (máximo 6) & Total & 0 \\
\hline
\end{tabular}

\begin{tabular}{|c|c|c|c|}
\hline II. Productividad ( $60 \%$ ) (se considera solo los últimos 5 años) & Puntos & Frc. IR & Puntaje IR \\
\hline \multicolumn{4}{|l|}{ Tem ( Se evalúan las coautorias según criterios fijados por los Grupos) } \\
\hline $\begin{array}{l}\text { ISI y revistas equivalentes ** (de acuerdo a criterios explícitos de equivalencias } \\
\text { determinados por cada Grupo de Estudio)* }\end{array}$ & 10 & & 0 \\
\hline Libro editorial con comité editorial, referato y amplia y reconocida participación & 10 & & 0 \\
\hline $\begin{array}{l}\text { Scielo y revistas equivalentes ( de acuerdo a criterios de equivalencia determinados } \\
\text { por cada Grupo de Estudio) }\end{array}$ & 7 & & 0 \\
\hline $\begin{array}{l}\text { Scielo y revistas equivalentes ( de acuerdo a criterios de equivalencia determinados } \\
\text { por cada Grupo de Estudio) }\end{array}$ & 5 & & 0 \\
\hline Capitulo libro editorial con Comité, referato, amplia y reconocida circulación & 5 & & 0 \\
\hline Revistas en otros índices (determinados por cada Grupo de Estudio)* & 3 & & 0 \\
\hline Capitulo libro editorial sin comité editorial y $\sin$ referato y de circulación restringida & 3 & & 0 \\
\hline Publicaciones en actas de congresos* & 2 & & 0 \\
\hline Traducciones y ediciones críticas de libros (casos filologia, filosofia e historia) & 5 & & 0 \\
\hline Total Productividad Investigador Responsable & & & 0 \\
\hline Total Capacidad + Productividad Investigador Responsable & & & 0 \\
\hline
\end{tabular}

Notas: La homogenización de los criterios de los grupos de Ciencias Sociales y Humanidades hacen necesario valorar tanto las revistas ISI y sus equivalentes, como libros de editoriales con comité editorial y referato, ya que hay diferencias entre las disciplinas respecto de los medios utilizados para comunicar los resultados de las investigaciones.

* Entre los criterios, deben considerarse, existencia de comité editorial, referato, antigüedad, continuidad y circulación.

** Los grupos, según los criterios y prácticas de cada una de las disciplinas, podrán diferenciar entre libros de editorial con comité editorial y referato, de artículos en revistas ISI. Si así lo hicieran, darán 12 a la máxima categoría y 10 puntos a las siguientes.

${ }^{33}$ Concurso FONDECYT Regular 2008 - Pauta de evaluación curricular Ciencias Sociales y Humanidades. Disponible en: <http://www.fondecyt.cl/578/article-27531.html>. [Consultado: el 8 de Febrero de 2008]. 